\title{
NON-DESTRUCTIVE TEMPERATURE DISTRIBUTION MEASUREMENT OF HIGH VISCOSITY FLOW OF USING ELECTRICAL CAPACITANCE TOMOGRAPHY
}

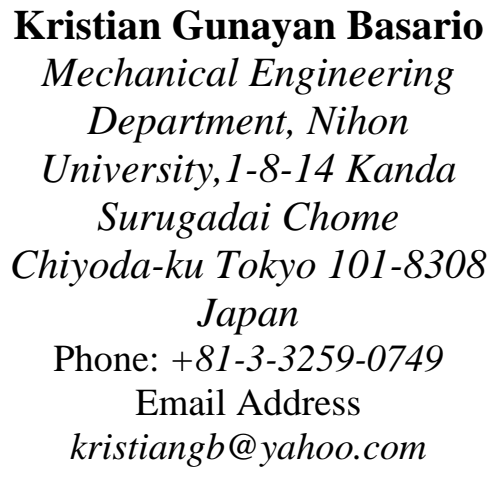

\author{
Je-Eun Choi \\ Mechanical Engineering \\ Department, Nihon \\ University,1-8-14 Kanda \\ Surugadai Chome \\ Chiyoda-ku Tokyo \\ 101-8308 Japan \\ Phone: +81-3-3259-0749 \\ Email Address \\ doolylove4@hotmail.com
}

\author{
Masahiro Takei \\ Mechanical Engineering \\ Department, Nihon \\ University,1-8-14 Kanda \\ Surugadai Chome \\ Chiyoda-ku Tokyo \\ 101-8308 Japan \\ Phone: +81-3-3259-0749 \\ Email Address \\ masa@mech.cst.nihon-u.ac.jp
}

Keywords: Polycarbonate resins, Temperature distribution, Relative permittivity, Electrical Capacitance Tomography, High Viscosity flow.

\begin{abstract}
Some of the problems in plastic industries are the rigidity and strength of the plastic products that cause from uneven temperature distribution especially that produced from the polycarbonate materials. This Polycarbonate material requires enough temperature in order to overcome the high viscosity during the process. This work presents the performance of the Electrical Capacitance Tomography (ECT) and its application in determining temperature distribution over high viscosity. ECT is designed to investigate measures and visualize inner process in a non-invasive and non-intrusive way. The main idea of ECT technique is collecting the cross section images within the area of the process container based on the capacitance measurement in which out of it the relative permittivity values are obtained. The polycarbonate resins were placed inside the ceramic container with 12-electrode sensors arranged equidistantly around the periphery of the container while the other ends are connected to ECT. Heat is evenly supplied with a specific period of time. The behavior of relative permittivity on the polycarbonate material due to heat effect is studied. Graphs are presented that depict the thermal characteristics and relative permittivity of the resins during phase change and thus found out that the temperature is a function of relative permittivity. The calculated value is the amount of heat penetrated that causes physical changes and the reconstructed images display the level of heat intensity occurs to the resins. The ECT can be used as a new technique in measuring temperature distribution.
\end{abstract}

\section{INTRODUCTION}

As the number of technological application for polymer increases, the study of their surface structure and material properties becomes more important (Dinelli, F., et al., 2001). In a very aggressive environment, industrial processes are characterized and operate by high temperature. It should be noted that many mass production manufacturing processes are operated at elevated temperatures which includes polymer extrusion (Jen, C.K., 2000). Polymers (especially during reprocessing) during injection molding may undergo thermal, thermo-oxidative and mechano-chemical degradation (Perez, J.M., et al., 2010) and hence the polymer properties may deteriorate significantly at high temperatures (Eguiazabal, J.I., et al., 1989). Polymer particularly Polycarbonate (PC) properties under heating process are need to monitor during operations to meet material performance and durability requirements and to avoid materials individual components degradation hence will lead in producing high quality products from efficient and less cost-effective technologies, and to attain these Electrical Capacitance Tomography has been introduced.

Electrical Capacitance Tomography (ECT) is one of the most promising tomography technique and was developed during late 1980s (Zhang, L.F., et al., 2009). It is a technique aimed to visualize the solid behavior in multiphase phase flow (Dyakowski, T., 1999, Halow, J.S., 1992) and imaging the spatial dielectric permittivity distribution of the materials of which they are made (Smolik, W. T., et al., 2009). The dielectric permittivity distribution images can be obtained from the capacitances value measured between each pair of electrodes (Liu S., et al., 2001) mounted circumferentially 
around the pipe.

This system has a low construction cost, it operates under high speed capability with high safety (Warsito, W., et al., 2001). Due to its robustness and small failure probability, ECT is suitable under harsh environmental operation condition (Watzenig, D., et al., 2009). And because of its being non-intrusive and non-invasive in nature, ECT system for a quantitative measurement has been well established (Mohamad, S. J., et al., 2002) and currently used in various fields.

There are two ways in achieving the purpose and computing the results of this technique: The forward problem using iterative method and the inverse problem using non-iterative method. Several conventional iterative and non-iterative methods has already been developed for more than a decade and used for different application processes. It was found out from previous studies (Yang. W.Q., et al., 2003) that, time consuming process and accuracy is the key issue of this both two methods for solving ill posed inverse problems; it is because certain numbers of iterations are necessary to get the minimum value of the result data, and it implies that the more iterations, the more errors produced and the longer period of time consumed. However, since there are only few algorithms commonly used for ECT systems that are available for the calculations, choices are depends on the application for there is no general - purpose algorithm (Dyakowski, T., et al., 1997).

Melting temperature $T_{m}$ is one of the temperature parameters of PC. $T_{m}$ is the temperature where in the whole polycarbonate is in the liquid phase (approx. $267^{\circ} \mathrm{C}$ and above). Below $T_{m}$ the material contains crystalline structure that contributes strength and rigidity, however, above $T_{m}$ the viscosity of the material gradually decreases without any distinct phase change. In this case its strength and rigidity were lost. Thus, proper temperature distribution and heat supply settings are necessary.

This paper discuss the on-line monitoring of temperature distribution during melting of high viscosity Polycarbonate resins (PC) using the non-destructive Electrical Capacitance Tomography (ECT) technique for a possible injection molding application. During the present study, the changes in electrical properties of polycarbonate due to increasing temperature have been explored. The electrical property such as relative permittivity has been determined from the gathered capacitances obtained by ECT. Out of determined relative permittivity, temperature distribution has been calculated using the govern equation. The main objective is to reconstruct phase transition images of PC and measures temperature distribution using ECT which will be comparing and validated from the results using Thermocouples.

\section{INTRODUCTION TO INJECTION MOLDING PROCESS}

Injection molding is one of the most widely used as plastic forming process in which plastic pellets are molten and injected into a mold of desired shape (Wong, H.Y., et al., 2008). According to (Belofsky, H.,1995, Gogos, C.G., \& Tadmor, Z., 1979, Middleman, S., 1977) the aim of injection molding machine is to produce high quality plastic parts, on time and with the highest level of profitability. More than one third of thermoplastic materials, such as TV case, mobile phones and CDs are processed with this technique because of its high- production features and low-cost (Osswald, T. A., et al., 2001), Furthermore this technique is used to manufacture also even in a small range of products, like micro-parts for implants (cochlear implants or cardiac sensors) and for medical equipment (syringe pumps and needles). For every application, it is sometimes difficult to achieve the quality of the finished parts which is critical even with well-controlled and accurately monitored parameters and materials (Ghita, O. R., et al., 2008) because the optimization of the injection molding machine is not a static process that can carried out only for once but rather it is dynamic continuous process and from (Offergeld, H., et al., 1992), the occurring faults and the continuous change of the ambient conditions, material and mold impose a constant re-examination of the process. Hence the process control and optimal parameter settings of product properties in injection molding processes requires accuracy (Chen, W. C., et al., 2008) because when the parameter settings are correct and accurate, the goal of the process and an efficient optimization can be obtained. Figure 1 below show the injection molding machine. Its operations are explained from (Vagelatos, G. A., et al., 2001).

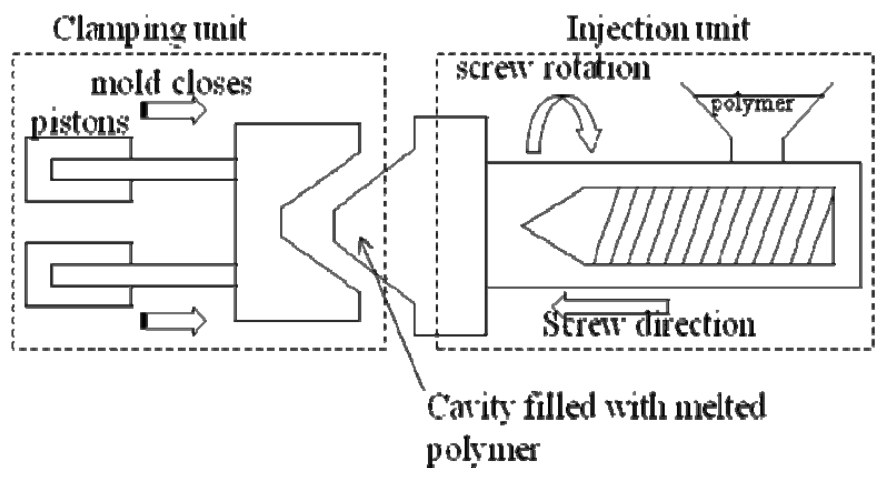

Figure 1. THE INJECTION MOLDING MACHINE

\section{TEMPERATURE-RELATIVE EQUATION RELATION}

The relative permittivity or dielectric constant $\varepsilon_{r}$ is one of the important molecular properties which can be useful predictor of other electrical properties of polymers (Bicerano, J., 1996) and is a function of so many parameters such as temperature (Liu, A., et al., 2007).

Relative permittivity or dielectric constant of a dielectric material is defined as;

$$
\varepsilon_{r}=\frac{\varepsilon}{\varepsilon_{0}}
$$

Where $\varepsilon$ is the amount of the charge that the plates carry in the presence of dielectric material when the plate is subject to potential difference. $\varepsilon_{0}$ is the amount of charge that the plates carry in the absence of dielectric material when the plate is subject to a potential difference. It is a constant permittivity of vacuum that describe the ability of material to store or hold electrostatic energy equal to $8.854 \times 10^{-12} \mathrm{~F} / \mathrm{m}$.

$$
\varepsilon=\varepsilon_{0}+P
$$


Thus;

$$
\varepsilon_{r}=\frac{\varepsilon_{0}+P}{\varepsilon_{0}}
$$

In the absence of dielectric material;

$$
\begin{gathered}
\varepsilon_{0}=\varepsilon_{0} E \\
\varepsilon_{r}=\frac{\varepsilon_{0} E+P}{\varepsilon_{0} E} \\
P=\varepsilon_{0} E\left(\varepsilon_{r}-1\right)
\end{gathered}
$$

The dielectric constant $\varepsilon_{r}$ is related to the molar polarization Pm using Debye equation;

$$
\frac{\varepsilon_{r}-1}{\varepsilon_{r}+2}=\frac{\rho P_{m}}{M}
$$

where:

$$
P_{m}=\frac{N_{A}}{3 \varepsilon_{0}}\left(\alpha_{e}+\frac{\mu^{2}}{3 k_{B} T}\right)
$$

By substituting Eq. 8 to Eq. 7 where temperature can be calculated will give;

$$
\frac{\varepsilon_{r}-1}{\varepsilon_{r}+2}=\frac{\rho}{M} \frac{N_{A}}{3 \varepsilon_{0}}\left(\alpha_{e}+\frac{\mu^{2}}{3 k_{B} T}\right) \quad \text { Eq. } 9
$$

where $\rho$ is the density $=1220 \mathrm{~kg} / \mathrm{m}^{3}, N_{A}$ is the Avogadro's number $=6.022137 \times 10^{23}, M$ is the molecular weight of the polycarbonate resins calculated theoretically in $\mathrm{kg} / \mathrm{mol}, \alpha_{e}$ is the electric/distortional polarizability in F-m ${ }^{2}$. It is the induced moment due to elastic polarization of the atom or molecule by the impressed field (Van Vleck, J.H., 1927 ) and in this case it is neglected due to the absence of electric field, $k_{B}$ is the Boltzmann constant $=1.38065 \mathrm{x}$ $10^{-23} \mathrm{~J} / \mathrm{K}, \mu$ is the permanent dipole moment $=5.004 \times 10^{-30}$ in Coulomb-meter (Fabre. M.J., et al., 1992), and $T$ is the absolute temperature.

\section{ECT GOVERNING EQUATION}

Giving emphasis in using inverse problem, mathematical equation source must be define clearly in order to manipulate and formulate new equation out of it that will suit to use in every algorithm. Since it is inverse, capacitance-permittivity relationship in a static-electro field is the source equation and can be expressed by:

$$
C_{i, j}=-\frac{\varepsilon_{0}}{V_{c}} \oint_{r \subseteq \Gamma_{j}} \varepsilon(\mathbf{r}) \nabla V_{i}(\mathbf{r}) \cdot d \mathbf{r}
$$

where $i$ is the standard electrode number that ranges from 1 to 11 , and $j$ is the reference electrode number, which ranges from $i+1$ to 12 . Ci,j is the measured capacitance between the standard electrode $i$ and the reference electrode $j, \varepsilon 0$ is the known vacuum permittivity, $\varepsilon(\mathbf{r})$ is the relative permittivity distribution on the cross section, $\mathbf{r}$ is a position vector on the cross section: $\mathbf{r}=(x, y), V c$ is the known voltage to the $i$ th electrode, $\_j$ is the area affected by the electric line of force and $\operatorname{Vi}(\mathbf{r})$ is the potential distribution on the cross section between the $i$ th and $j$ th electrodes. Even though the values of $\varepsilon(\mathbf{r})$ and $\operatorname{Vi}(\mathbf{r})$ in equation (10) are unknown, $\varepsilon(\mathbf{r})$ can be approximated by assuming that the electric charge's linear coupling at a position $\mathbf{r}$ with a weight of sensitivity in $\_j$ area is the total capacitance (Yang, W.Q., 1996). From previous work (Polydorides, N., et al., 2002), when a particle exists solely in a infinitely small $\Delta x \times \Delta y$ area at the center point $\mathrm{r}=\mathrm{r}_{0}$ between the $i$ and $j$ electrodes, and air exists at the remaining positions, the Laplace equation;

$$
\nabla \cdot\left[\varepsilon\left(\mathbf{r}_{0}\right) \nabla V\left(\mathbf{r}_{0}\right)\right]=0
$$

is assumed to hold in the cross section. A Finite Element Method can be used to discritize Eq. (11), and the distribution of $V_{i}(\mathrm{r})$ can be obtained by substituting the boundary conditions into Eq. (10). From (Choi JE, et al., 2010), the capacitance value $C_{i, j}\left(\mathbf{r}_{0}\right)$ is calculated when a particle is located at $\mathbf{r}=\mathbf{r}_{\mathbf{0}}$ from Eq.(10). The sensitivity value for a particle at $\mathbf{r}=\mathbf{r}_{\mathbf{0}}$ between standard electrode $i$ and the reference electrode $j$ is calculated using;

$$
S_{i j}\left(\mathbf{r}_{\mathbf{0}}\right)=\frac{c_{i j}\left(\mathbf{r}_{\mathbf{0}}\right)-{c_{i j}}^{\text {air }}}{{c_{i j}}^{\text {particle }}-{c_{i j}}^{\text {air }}}
$$

where $c_{i j}{ }^{a i r}$ and $c_{i j}{ }^{\text {particle }}$ are the capacitances when the static uniformly dispersed polycarbonate resins for calibration and the air occupy inside the ceramic container, respectively. Using Eq. 11 and Eq. 10, the sensitivity value are repeatedly obtained at every position $\mathbf{r}$ and for electrode pair between 1 and 2, through 1 and 7, considering for symmetrical structure. The linear coupling of normalized $s_{i j}(\mathbf{r})$ and $\varepsilon_{n}(\mathbf{r})$ is expressed by the normalized $c_{i j}$, that is

$$
c_{i j}=s_{i j} \cdot \varepsilon_{n}
$$

Where $\varepsilon_{n}=\left[\varepsilon(1,1), \varepsilon(1,2), \ldots, \varepsilon\left(n_{x}, n_{y}\right)\right]$ and $s_{i j}=\left[s_{i j}(1,1)\right.$, $\left.s_{i j}(1,2), \ldots, s_{i j}\left(n_{x}, n_{y}\right)\right]$.The above equation can be rewritten as the following matrix expression;

$$
\mathbf{C}=\mathbf{S}_{\mathbf{e}} \mathbf{E}
$$

Eq. 14

In other words, the capacitance CT can be used to obtain the permittivity distribution of particles $\mathbf{E}$ in the cross section from both the known sensitivity map matrix $\mathbf{S}_{\mathbf{e}}$ and the measured capacitance matrix $\mathbf{C}$. In the case of 12 electrodes and $32 \times 32=1024$ pixels, the sensitivity matrix $\mathbf{S}_{\mathbf{e}}$ in Eq. (14) is a $66 \times 1024$ matrix, the capacitance matrix $\mathbf{C}$ is a $66 \times 1$ column vector, and the permittivity distribution matrix $\mathbf{E}$ is a $1024 \times 1$ column vector. The mathematical method used to obtain the permittivity matrix $\mathbf{E}$ from both the capacitance matrix $\mathbf{C}$ and the sensitivity matrix $\mathbf{S}_{\mathbf{e}}$ is an ill-posed inverse problem because the inverse matrix $\mathbf{S}_{\mathbf{e}}{ }^{-1}$ does not exist. The raw values of $\mathbf{S}_{\mathbf{e}}$ have an extremely wide range; the sensitivity to an adjacent electrode pair is more than 100 times larger than that to an opposing electrode pair. 


\section{EXPERIMENT}

\subsection{Materials and Equipment}

A virgin commercial grade polycarbonate (PC) resins were used. It is said to be a promising polymeric material which posses a lot of desirable properties for electronic and optoelectronic applications (Radwan, R.M., et al., 2008) and one of the fastest growing engineering polymers notable for its high impact resistance and ductility over a broad temperature range (Bozi, J., et al., 2007, Mpoukouvalas, K., et al., 2006).

The samples of $1200 \mathrm{ml}$ PC were placed inside the ceramic circular container $(9.8 \mathrm{~cm}$ in dia.). This ceramic container is circumferentially mounted with 12-electrodes sensors connected to the ECT.

ECT system used input channels of the Data Acquisition Module 200E Capacitance measurements Unit (DAM 200E CMU from Process Tomography Ltd.) which contain CMOS electronic circuitry and a current integration amplifier. The system can accept voltages from $90-240 \mathrm{~V}, 50$ or $60 \mathrm{~Hz}$ frequency.

Heater is used as a heat source in melting the PC resins inside the ceramic container, and to measure the temperature directly and that will validate the calculated temperature from ECT, four (4) thermocouples (type K) has been inserted arranged with a certain distance within the radius of the said container. This thermocouples are attached to data acquisition/ data logger switch unit (AGILENT 34970A) to register the direct reading.

\subsection{Set up and methods}

Figure 1 below shows the complete set up of the experiment. In data collection process, capacitance values measured from the different parts of cross section are collected (by ECT) through the different pairs of 12-electrode system (Huang, S.M., et al., 1989). In permittivity image, a square grid of 32 x 32 pixels format are used to display (through computer) the distribution of the normalized composite permittivity of each pixel (Hunt, A., 2004).

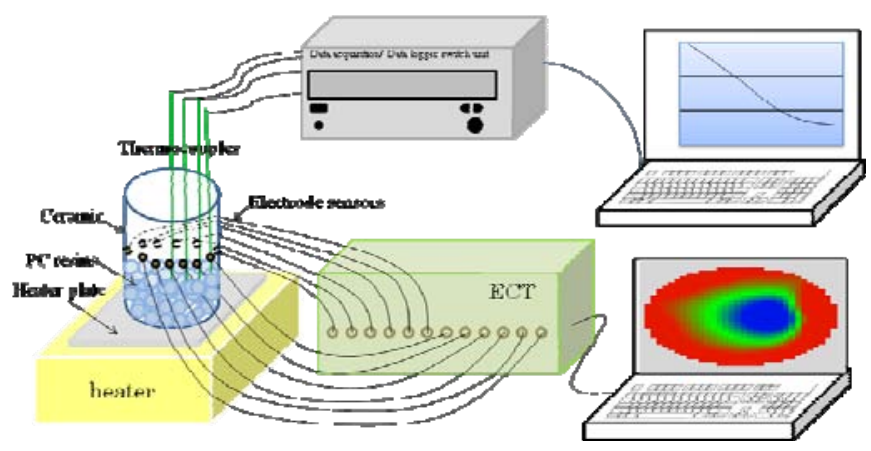

Figure 2. TRACKING OF TEMPERATURE DISTRIBUTION USING THERMOCOUPLES AND ECT WITH 12 ELCTRODES ARRAY

The normalized permittivity has that been collected by ECT will be converted to relative permittivity through normalization hence; will use to calculate the temperature as described from the equation 9.

\subsection{Condition}

It was assume that the center area of the heater has a high temperature value. During heating, the data from thermocouples and ECT were gathered simultaneously with a 10 minutes time allotted.

\subsection{Results}

Figure 3 shows the reconstructed images results using ECT. Images are time base calculation. Three (3) different color schemes are can be observe; red, green and blue colors. These changes of colors represents gradual physical transition and heat intensity to the polycarbonate resins as heating process occurs.

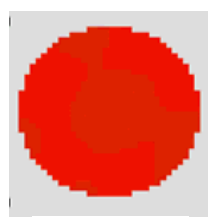

0 sec

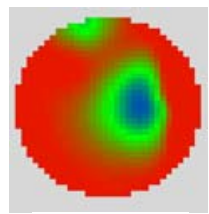

$240 \mathrm{sec}$

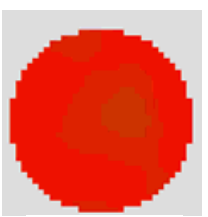

$60 \mathrm{sec}$

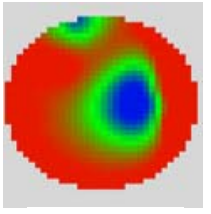

300 sec

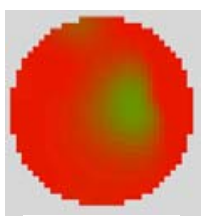

120 sec

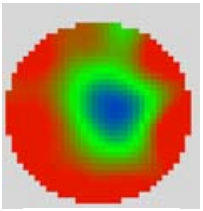

360 sec

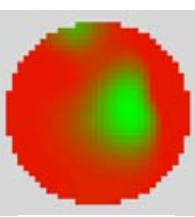

180 sec

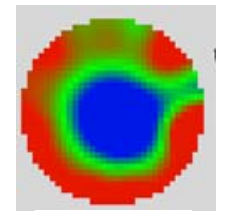

420 sec
Figure 3. TEMPERATURE DISTRIBUTION IMAGES DURING HEATING OF PC RESINS

The red color represents the dominated volume of solid polycarbonate resins inside the ceramic container. As heating process begins, it can be observed from 0 second to 180 seconds from being red it turns into green color. This shows that the PC gradually started to melt and changes its phase. As time elapsed and as it goes further heating, the heat saturates the center region in where the temperature is high at this point. It can be seen from 240 seconds to 420 seconds images that the green color turns to blue. This blue color represents melting temperature $T_{m}$ of PC. In this point the $\mathrm{PC}$ resins are in liquid phase. Due to too much heat penetrated to the inner molecules of PC, the molecular bonding and the chemical orientation of the atoms as being solid break and dispersed. Reaching this condition, the resins becomes less dense, low viscosity, its chemical constituents were lost and its possessed dielectric constant decreases.

\section{DISCUSSIONS}

Concerning relative permittivity, Figure 4 shows the normalized relative permittivity values of polycarbonate resins during heating. The lines are sloping in an upward right direction indicates that the relative permittivity value is lower at the center point (radius 0) and higher at the wall side area and as time elapse the relative permittivity values continually drop. This reveals that the relative permittivity vanishes dramatically (from center to wall) as phase transition occurs. 


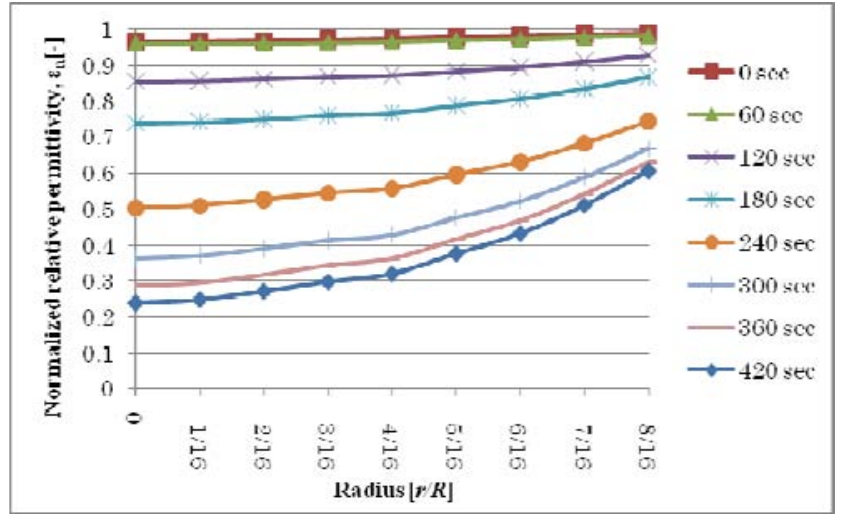

Figure 4. RELATIVE PERMITTIVITY DISTRIBUTION DURING HEATING OF PC RESINS

From the obtained relative permittivity distribution as shown from figure 4, temperature has been calculated. Experimentally, figure 5 shows the normalized amount of temperature in every corresponding relative permittivity in a particular point radius. By this much values the polycarbonate resins has been affected and phase transition has been occurred.

Furthermore, in this figure, it can be observed that the temperature at the centre point ( 0 radius) is highest and lowest at wall side point. Its slope indicates that the heat is penetrating towards the more solid and colder resins. Since heat is time dependent, the more time the more temperature becomes high and the resins becomes more liquid.

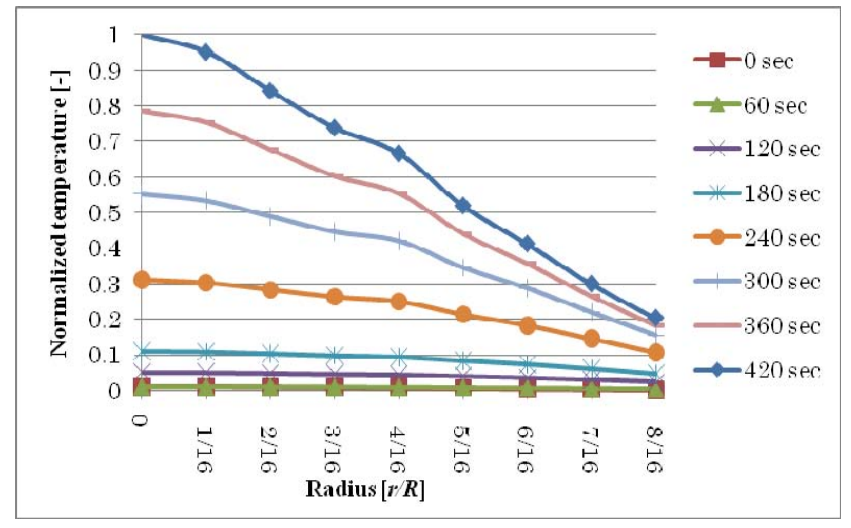

Figure 5. ECT TEMPERATURE DISTRIBUTION RESULTS DURING HEATING OF P C RESINS

Fig. 6 below shows the results of temperature distribution using thermocouples. The thermocouples read directly the temperature of the polycarbonate resins as the heat starts generated. The graph shows the temperature is highest at the centre of the container (radius 0 ) and lowest at the wall side region. As the lines go further away from the centre, temperature values abate. This figure validates figure 5 as far as the line behavior and the sequence of the temperature lines are concern. Both results show the same temperature line characteristics that display the same path of the temperature as it is distributed during melting of polycarbonate resins.

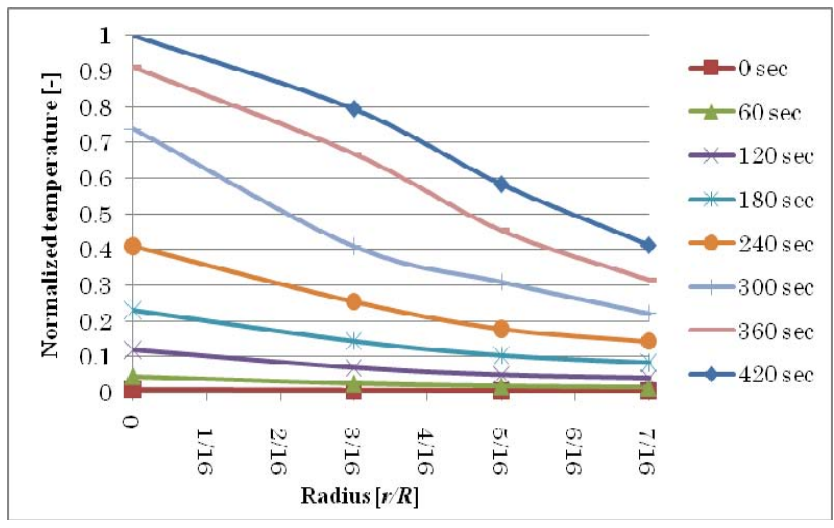

Figure 6. THERMOCOUPLE TEMPERATURE DISTRIBUTION RESULTS DURING HEATING OF PC RESINS

The experimental information or techniques in determining Temperature - Relative permittivity relation in a polymer particularly in polycarbonate resins are of much practical and academic interest in this study. Figure 7 below shows how the two parameters are inter-related. The curve of the graph illustrates and explains the phenomenon that as the temperature increases the relative permittivity dwindles.

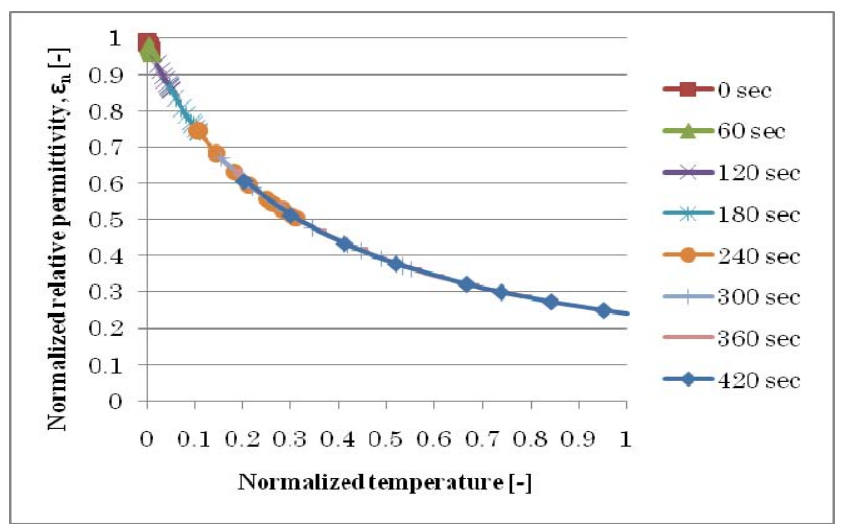

Figure 7. RELATIVE PERMITTIVITY-TEMPERATURE RELATION IN POLYCARBONATE PHASE TRANSITION

\section{CONCLUSIONS}

Based from the findings and analysis done to this study, the following conclusions can be drawn:

1. The results of using ECT are confirmed and validated from the thermocouples results. As can be observed, both show the same temperature line behavior. This implies that ECT is capable not only in measuring electrical properties particularly relative permittivity of any fluid medium such as polycarbonate materials but also it is applicable technique in tracking and measuring temperature distribution as proven from this study.

2. Using ECT, the relative permittivity as a macroscopic property of polycarbonate resins has been determined as a function of temperature. The graph shows that the relative permittivity decreases due to increasing temperature. This leads in bringing out to the understanding that the relative permittivity is inversely proportional to the temperature as

Copyright (C) 2011 by JSME 
shown from the result in fig. 7.

3. The reconstructed images produced from ECT shows the exact position of phase transition occurs to a high viscosity PC resins. It displays the heat intensity level of PC upon melting, and because of its being non-destructive in nature, ECT can produce visible images even in a high working temperature.

\section{REFERENCES}

Dinelli, F., et al., 2001, Thin Solid Films., Vol. 396, pp 138-144.

Jen, C.K., 2000, NDT\&E International., Vol. 33, pp . 145-153.

Perez, J.M., et al., 2010, Journal of Material Processing Technology., Vol. 210, pp 727-733.

Eguiazabal, J.I., et al., 1989, Eur. Polym. J., Vol. 25, pp 233-242.

Zhang, L.F., et al., 2009, Meas. Sci. Technol., Vol. 20, pp 1-7.

Dyakowski, T., 1999, Powder technology., Vol. 104, pp 287-295.

Halow, J.S., 1992, Powder Technology., Vol. 69, pp 255-277.

Smolik, W. T., et al., 2009, Meas. Sci. Technol., Vol. 20, pp $1-12$.

Liu S., et al., 2001, Meas. Sci. Technol. Vol. 12, pp 1120-112

Warsito, W., et al., 2001, Meas. Sci. Technol. Vol. 12, pp 2198-2210.

Watzenig, D., et al., 2009, Meas. Sci. Technol., Vol. 20, pp $1-22$.

Mohamad, S. J., et al., 2002, Meas. Sci. Tech., Vol. 13, pp1815-1821.

Yang. W.Q., et al., 2003, Meas. Sci. Technol., Vol. 14, pp R1-13.

Dyakowski, T., et al., 1997, Chemical Engineering Science, Vol. 52, pp 2099-2110

Wong, H.Y., et al., 2008, Sensors and Actuators A Vol. 141, pp 712-722

Belofsky, H., 1995, Plastics, New York : Hanser Publishers

Gogos, C.G., \& Tadmor, Z., (1979), Principles of Polymer Processing, New York: John Wiley \& Sons

Middleman, S., 1977, Fundamentals of Polymer Processing, New York: McGraw-Hill

Osswald, T. A., et al., 2001, Injection molding handbook, Hanser Publishers

Ghita, O. R., et al., 2008, Polymer Testing Vol. 27, pp 459-469

Offergeld, H., et al., 1992, Kunststoffe Vol. 82, pp 636-640

Chen, W. C., et al., 2008, Expert System with applications, Vol. 35, pp 843-849

Vagelatos, G. A., et al., 2001, Expert System with applications, Vol. 20, pp 207-216

Bicerano. J., 1996, Prediction of polymers properties. $2^{\text {nd }}$ ed. New York, Marcel dekker, pp 287

Liu, A., et al., 2007, Eur. Polym. J., Vol. 43, pp 989-995

Van Vleck, J.H., 1927, Physical Review., Vol. 29, pp 727-744

Fabre. M.J., et al., 1992, Polymer, Vol. 33, pp 194-197

Yang, W.Q., 1996, Meas. Sci. Tech. Vol. 7, pp 225-232

Polydorides, N., et al., 2002, Meas. Sci.Tech., Vol. 13, pp 1871-1883
Choi JE, et al., 2010, J. Fluid Sci. Tech., Vol. 00, pp 1-13

Radwan, R.M., et al., 2008, Nucl. Instr. and Meth. in Phys. Res., Vol. 226, pp 3588-3594

Bozi, J., et al., 2007, J. Anal. Appl. Pyrolisis., Vol. 79, pp 337-345

Mpoukouvalas, K., et al., 2006, Polymer., Vol. 47, pp 7170-7177

Huang, S.M., et al., 1989, J. Physics E, Vol. 22, pp 173-177 Hunt, A., 2004, Proceedings of ESDA $7^{\text {th }}$ Biennial ASME, pp 1-8 